\title{
Construction of Complex Contact Manifolds via Reduction
}

\author{
Mitsuhiro IMADA \\ Keio University \\ (Communicated by K. Ahara)
}

\begin{abstract}
Kobayashi [13] introduced complex contact manifolds as a variant of real contact manifolds. Later, Ishihara and Konishi [11] defined normality of complex contact manifolds as for Sasakian manifolds in real contact geometry. In this paper, we construct normal complex contact manifolds via reduction from hyperkähler manifolds, and give a new example of normal complex contact manifolds. To check the normality for the new examples, we give a useful identity about sectional curvatures of normal complex contact manifolds. We also give an explicit example of a non-normal complex almost contact metric structure on $S^{4 m+3} \times S^{4 n+3}$.
\end{abstract}

\section{Introduction}

The theory of complex contact geometry started with the papers of Kobayashi [13] and Boothby [4], [5], as a variant of real contact geometry. More recent examples, including complex projective space and the complex Heisenberg group, are given in [1] and [3]. Ishihara and Konishi [11] defined the so-called I-K normality of complex contact manifolds as for Sasakian manifolds in real contact geometry. In this paper, we construct normal complex contact manifolds via reduction from hyperkähler manifolds. Leaving the detailed notion of hyperkähler manifolds to Definition 5.1, we state the first main result as follows:

THEOREM A (Theorem 5.2). Let $\left(\tilde{M}, J_{1}, J_{2}, J_{3}, \tilde{g}\right)$ be a hyperkähler manifold. Assume that $\mathbf{C}^{*}$ acts on $\tilde{M}$ holomorphically, properly and freely. Then the quotient space $\widetilde{M} \backslash \mathbf{C}^{*}$ is naturally equipped with a smooth manifold structure and the quotient map $\pi: \widetilde{M} \longrightarrow$ $\tilde{M} \backslash \mathbf{C}^{*}$ canonically induces an I-K normal complex almost contact metric structure on $\tilde{M} \backslash \mathbf{C}^{*}$.

Using this theorem, we construct a new example of a normal complex contact manifold (Example 6.2), a quotient space $M=\left(\mathbf{C}^{4} \backslash\left\{z_{1} z_{2} z_{3} z_{4}=0\right\}\right) / \mathbf{C}^{*}$, where $\mathbf{C}^{*}$ acts on $\mathbf{C}^{4} \backslash\{0\}$ by

$$
\lambda \cdot\left(z_{1}, z_{2}, z_{3}, z_{4}\right)=\left(\lambda z_{1}, \lambda z_{2}, \lambda^{-1} z_{3}, \lambda^{-1} z_{4}\right) \quad\left(\lambda \in \mathbf{C}^{*}\right) .
$$

$M$ is diffeomorphic to $\mathbf{C}^{3} \backslash\left\{w_{1} w_{2} w_{3}=0\right\}$. This is a new example of an I-K normal complex almost contact metric manifold.

Received September 11, 2013; revised November 25, 2013

Key words and phrases: Complex contact manifolds; 3-Sasakian manifolds; 53D10 
This paper is organized as follows. In Section 2, we recall the definition of complex contact manifolds, which is a pair consisting of a manifold and a covering which admits a holomorphic 1-form $\omega$. It is known that there exists a complex contact metric structure on any complex contact manifold. The complex (almost) contact metric structure looks like two (almost) contact metric structures which transform to each other via the fixed complex structure. In Section 3, we recall the notion of I-K normality defined by Ishihara and Konishi [11].

This normality implies any complex contact metric manifold is also a Kähler manifold. We prove that the sectional curvature $K$ of an I-K normal complex contact metric manifold has a special property:

Proposition (Proposition 3.6). On I-K normal complex contact manifolds, we have

$$
K(X, J X)+K(X, G X)+K(X, H X)=6,
$$

for any $X \in \mathcal{H}$, where $K(X, Y)$ is the sectional curvatures of the plane spanned by $\{X, Y\}$, and $G, H$ and $J$ are associated to the complex contact metric structure (see Definition 2.3).

By this theorem, we can check whether any complex contact structure has I-K normality. For example, the odd dimensional complex projective space with the Fubini-Study metric is an I-K normal complex contact metric manifold, but there are no other known examples.

On the other hand, Korkmaz [15] has introduced a different notion of normality. As we show in Section 4, the normality Korkmaz defines is weaker than I-K normality, and there are some manifolds which admit normal which Korkmaz defines complex contact metric structures. In Section 5, we prove Theorem 1 and use it to construct new I-K normal complex contact metric manifolds by a projection from a hyperkähler manifold. In Section 6, we give a new example of I-K normal complex almost contact metric manifolds.

The second main result is to construct complex almost contact metric structures. CalabiEckmann [6] proved that $S^{2 p+1} \times S^{2 q+1}$ admits a complex structure $J$. In section 7, we show that 3-Sasakian structures on $S^{4 m+3}$ and $S^{4 n+3}$ induce a non-normal complex almost complex metric structure on $S^{4 m+3} \times S^{4 n+3}$ with respect to $J$.

THEOREM B (Theorem 7.2). The complex almost contact metric structure $\left(G_{m, n}, H_{m, n}, J_{m, n}, u_{m, n}, v_{m, n}, U_{m, n}, V_{m, n}, g_{m, n}\right)$ on $S^{4 m+3} \times S^{4 n+3}$ given by (15), (16), (18), (19) and (21) is not I-K normal.

\section{Definitions}

We first recall the notion of complex contact metric manifolds [3].

Definition 2.1. Let $M$ be a complex manifold with $\operatorname{dim}_{\mathbf{C}} M=2 n+1$ and $J$ the complex structure on $M . M$ is called a complex contact manifold if there exists an open covering $\mathcal{U}=\left\{\mathcal{O}_{\lambda}\right\}$ of $M$ such that: 
1) On each $\mathcal{O}_{\lambda}$ there is a holomorphic 1-form $\omega_{\lambda}$ with $\omega_{\lambda} \wedge\left(d \omega_{\lambda}\right)^{n} \neq 0$ everywhere;

2) If $\mathcal{O}_{\lambda} \cap \mathcal{O}_{\mu} \neq \phi$, there is a nonvanishing holomorphic function $h_{\lambda \mu}$ on $\mathcal{O}_{\lambda} \cap \mathcal{O}_{\mu}$ such that

$$
\omega_{\lambda}=h_{\lambda \mu} \omega_{\mu} \text { in } \mathcal{O}_{\lambda} \cap \mathcal{O}_{\mu} .
$$

For each $\mathcal{O}_{\lambda}$, we define a distribution $\mathcal{H}_{\lambda}=\left\{X \in T \mathcal{O}_{\lambda} \mid \omega_{\lambda}(X)=0\right\}$. Note that the $h_{\lambda \mu}$ are nonvanishing, and $\mathcal{H}_{\lambda}=\mathcal{H}_{\mu}$ on $\mathcal{O}_{\lambda} \cap \mathcal{O}_{\mu}$. Thus $\mathcal{H}=\cup \mathcal{H}_{\lambda}$ is a holomorphic, nonintegrable subbundle on $M$, called the horizontal subbundle.

DeFinition 2.2. Let $M$ be a complex manifold with $\operatorname{dim}_{\mathbf{C}}=2 n+1$ and $J$ a complex structure. Let $g$ be a Hermitian metric. $M$ is called a complex almost contact metric manifold if there exists an open covering $\mathcal{U}=\left\{\mathcal{O}_{\lambda}\right\}$ of $M$ such that:

1) On each $\mathcal{O}_{\lambda}$ there are 1-forms $u_{\lambda}$ and $v_{\lambda}=u_{\lambda} J,(1,1)$ tensors $G_{\lambda}$ and $H_{\lambda}=G_{\lambda} J$, unit vector fields $U_{\lambda}$ and $V_{\lambda}=-J U_{\lambda}$ such that

$$
\begin{aligned}
& G_{\lambda} J_{\lambda}=-J_{\lambda} G_{\lambda}, \quad H_{\lambda}^{2}=G_{\lambda}^{2}=-i d+u_{\lambda} \otimes U_{\lambda}+v_{\lambda} \otimes V_{\lambda}, \\
& g\left(G_{\lambda} X, Y\right)=-g\left(X, G_{\lambda} Y\right), \quad g\left(U_{\lambda}, X\right)=u_{\lambda}(X), \\
& G_{\lambda} U_{\lambda}=0, \quad u_{\lambda}\left(U_{\lambda}\right)=1 ;
\end{aligned}
$$

2) If $\mathcal{O}_{\lambda} \cap \mathcal{O}_{\mu} \neq \phi$, there are functions $a, b$ on $\mathcal{O}_{\lambda} \cap \mathcal{O}_{\mu}$ such that

$$
\begin{aligned}
& u_{\mu}=a u_{\lambda}-b v_{\lambda}, \quad v_{\mu}=b u_{\lambda}+a v_{\lambda}, \\
& G_{\mu}=a G_{\lambda}-b H_{\lambda}, \quad H_{\mu}=b G_{\lambda}+a H_{\lambda}, \\
& a^{2}+b^{2}=1 .
\end{aligned}
$$

DEFINITION 2.3. Let $\left(M,\left\{\omega_{\lambda}\right\}\right)$ be a complex contact manifold with complex contact structure $J$ and Hermitian metric $g$. We call $(M, u, v, U, V, J, g)$ a complex contact metric manifold if there exists an open covering $\mathcal{U}=\left\{\mathcal{O}_{\lambda}\right\}$ of $M$ such that (here and below $G=G_{\lambda}$, etc) :

1) On each $\mathcal{O}_{\lambda}$ there is a local $(1,1)$ tensor $G_{\lambda}$ such that $\left(u_{\lambda}, v_{\lambda}, U_{\lambda}, V_{\lambda}, G_{\lambda}, H_{\lambda}=\right.$ $\left.G_{\lambda} J, g\right)$ is an almost contact metric structure on $M$;

2) $g\left(X, G_{\lambda} Y\right)=d u_{\lambda}(X, Y)+\left(\sigma_{\lambda} \wedge v_{\lambda}\right)(X, Y)$ and $g\left(X, H_{\lambda} Y\right)=d v_{\lambda}(X, Y)-\left(\sigma_{\lambda} \wedge\right.$ $\left.u_{\lambda}\right)(X, Y)$, where $\sigma_{\lambda}(X)=g\left(\nabla_{X} U_{\lambda}, V_{\lambda}\right)$ with $\nabla$ the Levi-Civita connection with respect to $g$.

REMARK 2.4. Foreman [8] showed the existence of complex contact metric structures on complex contact manifolds.

REMARK 2.5. We can locally choose orthonormal vectors $X_{1}, \ldots, X_{n}$ in $\mathcal{H}$ such that $\left\{X_{i}, J X_{i}, G X_{i}, H X_{i}, U, V \mid 1 \leq i \leq n\right\}$ is an orthonormal basis of the tangent spaces of $U_{\alpha}$. 


\section{I-K normality on complex contact structures}

We recall the definition of I-K normality introduced by Ishihara and Konishi [10] for (almost) complex contact metric structures. We set the two tensor fields $S$ and $T$ by,

$$
\begin{aligned}
S(X, Y)= & {[G, G](X, Y)+2 g(X, G Y) U-2 g(X, H Y) V } \\
& +2 v(Y) H X-2 v(X) H Y+\sigma(G Y) H X \\
& -\sigma(G X) H Y+\sigma(X) G H Y-\sigma(Y) G H X, \\
T(X, Y)= & {[H, H](X, Y)-2 g(X, G Y) U+2 g(X, H Y) V } \\
& +2 u(Y) G X-2 u(X) G Y+\sigma(H X) G Y \\
& -\sigma(H Y) G X+\sigma(X) G H Y-\sigma(Y) G H X .
\end{aligned}
$$

DEFinition 3.1. A complex contact manifold $M$ is I-K normal if the tensors $S$ and $T$ both vanish.

REMARK 3.2. I-K normality implies that the underlying Hermitian manifold $(M, J, g)$ is a Kähler manifold (cf. [11]).

We recall properties obtained by Korkmaz [15].

PROPOSITION 3.3. On an I-K normal complex contact manifold, for $X, Y, Z \in \mathcal{H}$, we have

$$
\begin{aligned}
g\left(\left(\nabla_{X} G\right) Y, Z\right)= & -\sigma(X) g(H X, Y)+v(X) \Omega(G Z, G Y) \\
& -2 v(X) g(H G Y, Z)-u(Y) g(X, Z)-v(Y) g(J X, Z) \\
& +u(Z) g(X, Y)-v(Z) g(X, J Y),
\end{aligned}
$$

and

$$
\begin{aligned}
g\left(\left(\nabla_{X} H\right) Y, Z\right)= & -\sigma(X) g(G X, Y)+u(X) \Omega(H Z, G H Y) \\
& -2 u(X) g(H G Y, Z)+u(Y) g(J X, Z)-v(Y) g(X, Z) \\
& +u(Z) g(X, J Y)+v(Z) g(X, Y),
\end{aligned}
$$

where $\nabla$ is the Levi-Civita connection with respect to $g$.

Lemma 3.4. Under the same assumptions as in Proposition 3.3, we have

$$
\begin{aligned}
g(R(X, G X) Y, G Y)= & g(R(X, Y) X, Y)+g(R(X, G Y) X, G Y) \\
& +4 g(J X, Y) \Omega(X, Y)-4 g(H X, Y) \Omega(G X, Y) \\
& -2 g(G X, Y)^{2}-4 g(H X, Y)^{2}-2 g(X, Y)^{2} \\
& +2 g(X, X) g(Y, Y)-4 g(J X, Y)^{2},
\end{aligned}
$$

and 


$$
\begin{aligned}
g(R(X, H X) Y, H Y)= & g(R(X, Y) X, Y)+g(R(X, H Y) X, H Y) \\
& +4 g(J X, Y) \Omega(X, Y)+4 g(G X, Y) \Omega(H X, Y) \\
& -2 g(H X, Y)^{2}-4 g(G X, Y)^{2}-2 g(X, Y)^{2} \\
& +2 g(X, X) g(Y, Y)-4 g(J X, Y)^{2} .
\end{aligned}
$$

LEMMA 3.5. On an I-K normal complex contact manifold, for $X \in \mathcal{H}$, we have

$$
\Omega(J X, X)=-2 g(X, X) .
$$

PROOF. Since $J$ is parallel for $\nabla$,

$$
\begin{aligned}
g(R(X, H X) J X, G X) & =g(J R(X, H X) X, J H X) \\
& =-g(R(X, H X) H X, X) .
\end{aligned}
$$

By Lemma 3.4, we get

$$
\begin{aligned}
g(R(X, H X) J X, G X)= & -g(R(X, H X) H X, X) \\
& -2 g(X, X)(\Omega(J X, X)+2 g(X, X)) .
\end{aligned}
$$

Comparing the right hand sides of (8) and (9), we get the lemma.

Finally, we have the following property of sectional curvatures.

PROPOSITION 3.6. On I-K normal complex contact manifolds, we have

$$
K(X, J X)+K(X, G X)+K(X, H X)=6,
$$

for any horizontal vector field $X$, where $K(X, Y)$ is the sectional curvature of the plane spanned by $\{X, Y\}$.

ProOF. Since $J$ is parallel for $\nabla$,

$$
\begin{aligned}
g(R(X, G X) J X, G J X)) & =-g(J R(X, G X) X, J G X) \\
& =g(R(X, G X) G X, X) .
\end{aligned}
$$

On the other hand, by Lemmas 3.4 and 3.5,

$$
\begin{aligned}
g(R(X, G X) J X, G J X)= & -g(R(X, J X) J X, X)-g(R(X, H X) H X, X) \\
& -4 g(X, X) \Omega(J X, X)-2 g(X, X)^{2} \\
= & -g(R(X, J X) J X, X)-g(R(X, H X) H X, X) \\
& +6 g(X, X)^{2} .
\end{aligned}
$$

This gives the conclusion.

EXAMPLES 3.7. The odd-dimensional complex projective space with the FubiniStudy metric of constant holomorphic curvature 4 is an example of an I-K normal complex contact structure and satisfies $K(X, G X)=K(X, H X)=1$ (see Example 6.1 below or [15]). 


\section{Normality of complex contact manifolds}

The notion of I-K normality seems too strong, since the complex Heisenberg group admits no I-K normal contact metric structure while the real Heisenberg group admits a normal contact metric structure. Korkmaz introduced a weaker version of normality as follows.

DEFINITION 4.1. A complex contact metric structure is normal in the sense of [15] if

$$
\begin{cases}S(X, Y)=T(X, Y)=0 & \text { for every } X, Y \in \mathcal{H}, \\ S(U, Y)=T(V, Y)=0 & \text { for every } Y\end{cases}
$$

From now on, we use this definition of normality.

The following lemma is obtained by Korkmaz [15] .

LEMMA 4.2. If $X$ is a horizontal vector field on a normal complex contact metric manifold, then

$$
\begin{array}{r}
g(R(X, J X) J X, X)+g(R(X, G X) G X, X)+g(R(X, H X) H X, X) \\
=-6 g(X, X)(\Omega(J X, X)+g(X, X)) .
\end{array}
$$

EXAMPLE 4.3. We introduce the example of the complex Heisenberg group, the closed subgroup $\mathbf{H}_{\mathbf{C}}$ of $G L(3, \mathbf{C})$ given by

$$
\left\{\left(\begin{array}{ccc}
1 & b_{12} & b_{13} \\
0 & 1 & b_{23} \\
0 & 0 & 1
\end{array}\right) \mid b_{12}, b_{13}, b_{23} \in \mathbf{C}\right\} .
$$

Blair [1] defined the following complex contact metric structure on $\mathbf{H}_{\mathbf{C}}$ (see also [3]). Let $z_{1}, z_{2}, z_{3}$ be the coordinates on $\mathbf{H}_{\mathbf{C}} \cong \mathbf{C}^{3}$, defined by $z_{1}(A)=b_{23}, z_{2}(A)=b_{12}, z_{3}(A)=b_{13}$ for $A$ in $\mathbf{H}_{\mathbf{C}}$. Then the Hermitian metric

$$
g=\frac{1}{8}\left(\begin{array}{ccc|ccc} 
& & & 1+\left|z_{2}\right|^{2} & 0 & -z_{2} \\
& 0 & & 1 & 0 \\
& & & -\bar{z}_{2} & 0 & 1 \\
\hline 1+\left|z_{2}\right|^{2} & 0 & -\bar{z}_{2} & & & \\
0 & 1 & 0 & & 0 & \\
-z_{2} & 0 & 1 & &
\end{array}\right)
$$

is a left invariant metric on $\mathbf{H}_{\mathbf{C}}$. We define real 1-forms $u, v$ and unit vector fields $U, V$ by decomposing the holomorphic 1-form $\theta=\left(d z_{3}-z_{2} d z_{1}\right) / 2$ and the complex vector field $X=4\left(\partial / \partial z_{3}\right)$ into their real parts and the imaginary parts:

$$
\theta=u-i v, \quad X=U+i V .
$$


Also define two type- $(1,1)$ tensors

$$
\begin{aligned}
G & =\left(\begin{array}{ccc|ccc} 
& & 0 & 1 & 0 \\
& 0 & & -1 & 0 & 0 \\
& & & 0 & z_{2} & 0 \\
\hline 0 & 1 & 0 & & & \\
-1 & 0 & 0 & & 0 & \\
0 & \bar{z}_{2} & 1 & & &
\end{array}\right), \\
H & =\left(\begin{array}{ccc|ccc} 
& & & 0 & -i & 0 \\
& 0 & & i & 0 & 0 \\
0 & i & 0 & & & \\
-i & 0 & 0 & & 0 & \\
0 & i \bar{z}_{2} & 1 & &
\end{array}\right) .
\end{aligned}
$$

Then one can check that $(u, v, U, V, G, H, J, g)$ is a normal complex contact metric structure on $\mathbf{H}_{\mathbf{C}}$ [15].

\section{I-K normal complex contact structures from hyperkähler structures}

In this section, we construct a normal complex contact structure on the quotient space of a hyperkähler manifold via a $\mathbf{C}^{*}$ action. We first recall the definition of hyperkähler manifolds.

Definition 5.1. ( $\left(M, J_{1}, J_{2}, J_{3}, g\right)$ is a hyperkähler manifold if $J_{1}, J_{2}, J_{3}$ are complex structures on a complex manifold $M$ satisfying

$$
J_{1}^{2}=J_{2}^{2}=J_{3}^{2}=J_{1} J_{2} J_{3}=-i d,
$$

and $g$ is a Hermitian metric on $M$ with respect to $J_{1}, J_{2}$ and $J_{3}$.

We can produce a complex contact metric manifold from hyperkähler manifolds

THEOREM 5.2. Let $\left(\tilde{M}, J_{1}, J_{2}, J_{3}, \tilde{g}\right)$ be a hyperkähler manifold. We assume that $\mathbf{C}^{*}$ acts on $\tilde{M}$ holomorphically, properly and freely. Then the quotient space $\tilde{M} \backslash \mathbf{C}^{*}$ is naturally equipped with a smooth manifold structure and the quotient map $\pi: \tilde{M} \longrightarrow \tilde{M} \backslash \mathbf{C}^{*}$ canonically induces an I-K normal complex almost contact metric structure on $\tilde{M} / \mathbf{C}^{*}$.

Proof. Let $\left\{\widetilde{O}_{\lambda}\right\}$ be an open covering of $\widetilde{M}$. We choose local sections $s_{\lambda}: \pi\left(\widetilde{O}_{\lambda}\right) \longrightarrow$ $\widetilde{O}_{\lambda}$. Then we define type $(1,1)$ tensors $J, G$ and $H$ on $T\left(\tilde{M} / \mathbf{C}^{*}\right)$, and 1 -forms $u, v$ by

$$
\left\{\begin{array}{l}
J_{1}\left(s_{\lambda}\right)_{*} X=\left(s_{\lambda}\right)_{*} J X \\
J_{2}\left(s_{\lambda}\right)_{*} X=\left(s_{\lambda}\right)_{*} G X+u(X) v+v(X) J_{1} v, \\
J_{3}\left(s_{\lambda}\right)_{*} X=\left(s_{\lambda}\right)_{*} H X-v(X) v+u(X) J_{1} v,
\end{array}\right.
$$


where $v$ and $J_{1} v$ are unit tangent vectors to the orbit by $\mathbf{C}^{*}$. For example, $u$ and $v$ are explicitly given by

$$
u(X)=\tilde{g}\left(J_{2} s_{*} X, v\right), \quad v(X)=\tilde{g}\left(J_{2} s_{*} X, J_{1} v\right)=-\tilde{g}\left(J_{3} s_{*} X, v\right) .
$$

Finally, we define the unit vector fields $U, V$ on $\tilde{M} / \mathbf{C}^{*}$ by

$$
U=-\pi_{*}\left(J_{2} v\right), \quad V=\pi_{*}\left(J_{3} v\right) .
$$

It is easily seen that the structure $(u, v, U, V, G, H, J, g)$ (with $g$ the metric induced by $\tilde{g}$ ) satisfies Definition 2.3 and Definition 3.1.

\section{Examples of I-K normal complex contact structures from hyperkähler struc- tures}

EXAMPLES 6.1. By Theorem 5.2, the complex projective space $\mathbf{C} P^{2 n+1}$ with the Fubini-Study metric admits an I-K normal complex contact structure. Now we express this structure analytically in the case of $n=1$.

Let $\mathbf{C}^{4} \backslash\{0\}$ have the hyperkähler structure $\left(J_{1}, J_{2}, J_{3},\langle\rangle,\right)$, where $J_{1}, J_{2}$ and $J_{3}$ act on the position vector $p=\left(z_{1}, z_{2}, z_{3}, z_{4}\right)$ by

$$
\begin{aligned}
& J_{1} p=\left(i z_{1}, i z_{2}, i z_{3}, i z_{4}\right), \\
& J_{2} p=\left(\bar{z}_{3}, \bar{z}_{4},-\bar{z}_{1},-\bar{z}_{2}\right), \\
& J_{3} p=\left(i \bar{z}_{3}, i \bar{z}_{4},-i \bar{z}_{1},-i \bar{z}_{2}\right),
\end{aligned}
$$

and $\langle$,$\rangle is the standard metric on \mathbf{C}^{4} \backslash\{0\}$. We denote the norm $\sqrt{\sum_{k=1}^{4} z_{k} \bar{z}_{k}}$ of $z=$ $\left(z_{1}, z_{2}, z_{3}, z_{4}\right)$ by $\|z\|$.

$\mathbf{C}^{*}$ acts on $\mathbf{C}^{4} \backslash\{0\}$ by $\lambda \cdot\left(z_{1}, z_{2}, z_{3}, z_{4}\right)=\left(\lambda z_{1}, \lambda z_{2}, \lambda z_{3}, \lambda z_{4}\right)$, which acts freely and commutes with $J_{1}$. We can easily check that at $z$, the orbit space of $\mathbf{C}^{*}$ has tangent space at $z$ spanned by vectors

$$
\begin{gathered}
v=\frac{1}{2\|z\|} \sum_{j=1}^{4}\left(z_{j} \frac{\partial}{\partial z_{j}}+\bar{z}_{j} \frac{\partial}{\partial \bar{z}_{j}}\right), \\
J_{1} v=\frac{i}{2\|z\|} \sum_{j=1}^{4}\left(z_{j} \frac{\partial}{\partial z_{j}}-\bar{z}_{j} \frac{\partial}{\partial \bar{z}_{j}}\right) .
\end{gathered}
$$

By calculating with the inner product $\langle\rangle,$,$u and v$ are given by

$$
u=\frac{1}{2\|z\|} \sum_{j=1}^{2}\left(-z_{2 j-1} d z_{2 j}-\bar{z}_{2 j-1} d \bar{z}_{2 j}+z_{2 j} d z_{2 j-1}+\bar{z}_{2 j} d \bar{z}_{2 j-1}\right)
$$




$$
v=\frac{-i}{2\|z\|} \sum_{j=1}^{2}\left(z_{2 j-1} d z_{2 j}-\bar{z}_{2 j-1} d \bar{z}_{2 j}-z_{2 j} d z_{2 j-1}+\bar{z}_{2 j} d \bar{z}_{2 j-1}\right) .
$$

Then in complex coordinates $G$ and $H$ are given by

$$
G=\frac{1}{\|z\|^{2}}\left(\begin{array}{cc}
O & A \\
\bar{A} & O
\end{array}\right), \quad H=\frac{1}{\|z\|^{2}}\left(\begin{array}{cc}
O & i A \\
-i \bar{A} & O
\end{array}\right),
$$

where

$$
A=i\left(\begin{array}{cccc}
z_{2} \bar{z}_{1} & -\|z\|^{2}+z_{2} \bar{z}_{2} & z_{2} \bar{z}_{3} & z_{2} \bar{z}_{4} \\
\|z\|^{2}-z_{1} \bar{z}_{1} & -z_{1} \bar{z}_{2} & -z_{1} \bar{z}_{3} & -z_{1} \bar{z}_{4} \\
z_{4} \bar{z}_{1} & z_{4} \bar{z}_{2} & z_{4} \bar{z}_{3} & -\|z\|^{2}+z_{4} \bar{z}_{4} \\
-z_{3} \bar{z}_{1} & -z_{3} \bar{z}_{2} & \|z\|^{2}-z_{3} \bar{z}_{3} & -z_{3} \bar{z}_{4}
\end{array}\right)
$$

Finally, the two vector fields $U$ and $V$ are given by

$$
\begin{aligned}
& U=\frac{1}{2\|z\|} \sum_{j=1}^{2}\left(-\bar{z}_{2 j-1} d z_{2 j}-z_{2 j-1} d \bar{z}_{2 j}+\bar{z}_{2 j} d z_{2 j-1}+z_{2 j} d \bar{z}_{2 j-1}\right), \\
& V=\frac{i}{2\|z\|} \sum_{j=1}^{2}\left(\bar{z}_{2 j-1} d z_{2 j}-z_{2 j-1} d \bar{z}_{2 j}-\bar{z}_{2 j} d z_{2 j-1}+z_{2 j} d \bar{z}_{2 j-1}\right) .
\end{aligned}
$$

With the Fubini-Study metric $g$, we find that this complex contact metric structure $(u, v, U, V, G, H, J, g)$ is I-K normal and satisfies Proposition 3.6.

By modifying the $\mathbf{C}^{*}$ action on $\mathbf{C}^{4} \backslash\{0\}$, we can give another example of a normal complex contact metric manifold.

EXAMPLES 6.2. We consider another $\mathbf{C}^{*}$ action on $\mathbf{C}^{4} \backslash\left\{z_{1} z_{2} z_{3} z_{4}=0\right\}$ by

$$
\lambda \cdot\left(z_{1}, z_{2}, z_{3}, z_{4}\right)=\left(\lambda z_{1}, \lambda z_{2}, \lambda^{-1} z_{3}, \lambda^{-1} z_{4}\right),
$$

which also acts freely and commutes with $J_{1}$. This orbit space has tangent spaces at $z$ spanned by the vectors

$$
\begin{array}{r}
v=\frac{1}{2\|z\|}\left(z_{1} \frac{\partial}{\partial z_{1}}+\bar{z}_{1} \frac{\partial}{\partial \bar{z}_{1}}+z_{2} \frac{\partial}{\partial z_{2}}+\bar{z}_{2} \frac{\partial}{\partial \bar{z}_{2}}\right. \\
\left.-z_{3} \frac{\partial}{\partial z_{3}}-\bar{z}_{3} \frac{\partial}{\partial \bar{z}_{3}}-z_{4} \frac{\partial}{\partial z_{4}}-\bar{z}_{4} \frac{\partial}{\partial \bar{z}_{4}}\right), \\
J_{1} v=\frac{i}{2\|z\|}\left(z_{1} \frac{\partial}{\partial z_{1}}-\bar{z}_{1} \frac{\partial}{\partial \bar{z}_{1}}+z_{2} \frac{\partial}{\partial z_{2}}-\bar{z}_{2} \frac{\partial}{\partial \bar{z}_{2}}\right. \\
\left.-z_{3} \frac{\partial}{\partial z_{3}}+\bar{z}_{3} \frac{\partial}{\partial \bar{z}_{3}}-z_{4} \frac{\partial}{\partial z_{4}}+\bar{z}_{4} \frac{\partial}{\partial \bar{z}_{4}}\right) .
\end{array}
$$


We check that the quotient space $M=\left(\mathbf{C}^{4} \backslash\left\{z_{1} z_{2} z_{3} z_{4}=0\right\}\right) / \mathbf{C}^{*}$ is a complex manifold. We define a biholomorphic map $F$ on $M$ by

$$
F\left(\left[z_{1}, z_{2}, z_{3}, z_{4}\right]\right)=\left(\frac{z_{2}}{z_{1}}, z_{1} z_{3}, z_{1} z_{4}\right) .
$$

This map shows that $M$ is diffeomorphic to $\mathbf{C}^{3} \backslash\left\{w_{1} w_{2} w_{3}=0\right\}$. By a direct computation of the standard inner product $\langle\rangle,$,$u and v$ are given by

$$
\begin{aligned}
u= & \frac{-i}{2\|z\|}\left(-z_{1} d z_{4}+\bar{z}_{1} d \bar{z}_{4}+z_{2} d z_{3}-\bar{z}_{2} d \bar{z}_{3}\right. \\
& \left.-z_{3} d z_{2}+\bar{z}_{3} d \bar{z}_{2}-z_{4} d z_{1}+\bar{z}_{4} d \bar{z}_{1}\right), \\
v= & \frac{1}{2\|z\|}\left(-z_{1} d z_{4}-\bar{z}_{1} d \bar{z}_{4}+z_{2} d z_{3}+\bar{z}_{2} d \bar{z}_{3}\right. \\
& \left.+z_{3} d z_{2}+\bar{z}_{3} d \bar{z}_{2}-z_{4} d z_{1}-\bar{z}_{4} d \bar{z}_{1}\right) .
\end{aligned}
$$

Then in complex coordinates $G$ and $H$ are given as follows:

$$
G=\frac{1}{\|z\|^{2}}\left(\begin{array}{cc}
O & A \\
\bar{A} & O
\end{array}\right), \quad H=\frac{1}{\|z\|^{2}}\left(\begin{array}{cc}
O & i A \\
-i \bar{A} & O
\end{array}\right),
$$

where

$$
A=i\left(\begin{array}{cccc}
z_{1} \bar{z}_{4} & -z_{1} \bar{z}_{3} & -z_{1} \bar{z}_{2} & -\|z\|^{2}+z_{1} \bar{z}_{1} \\
z_{2} \bar{z}_{4} & -z_{2} \bar{z}_{3} & \|z\|^{2}-z_{2} \bar{z}_{2} & z_{2} \bar{z}_{1} \\
-z_{3} \bar{z}_{4} & -\|z\|^{2}+z_{3} \bar{z}_{3} & z_{3} \bar{z}_{2} & -z_{3} \bar{z}_{1} \\
\|z\|^{2}-z_{4} \bar{z}_{4} & z_{4} \bar{z}_{3} & z_{4} \bar{z}_{2} & -z_{4} \bar{z}_{1}
\end{array}\right)
$$

Finally, the two vector fields $U$ and $V$ are given by

$$
\begin{aligned}
U= & \frac{i}{2\|z\|}\left(z_{1} \frac{\partial}{\partial \bar{z}_{4}}-\bar{z}_{1} \frac{\partial}{\partial z_{4}}-z_{2} \frac{\partial}{\partial \bar{z}_{3}}+\bar{z}_{2} \frac{\partial}{\partial z_{3}}\right. \\
& \left.-z_{3} \frac{\partial}{\partial \bar{z}_{2}}+\bar{z}_{3} \frac{\partial}{\partial z_{2}}+z_{4} \frac{\partial}{\partial \bar{z}_{1}}-\bar{z}_{4} \frac{\partial}{\partial z_{1}}\right), \\
V= & \frac{-1}{2\|z\|}\left(z_{1} \frac{\partial}{\partial \bar{z}_{4}}+\bar{z}_{1} \frac{\partial}{\partial z_{4}}-z_{2} \frac{\partial}{\partial \bar{z}_{3}}-\bar{z}_{2} \frac{\partial}{\partial z_{3}}\right. \\
& \left.-z_{3} \frac{\partial}{\partial \bar{z}_{2}}-\bar{z}_{3} \frac{\partial}{\partial z_{2}}+z_{4} \frac{\partial}{\partial \bar{z}_{1}}+\bar{z}_{4} \frac{\partial}{\partial z_{1}}\right) .
\end{aligned}
$$

With the induced metric $g$ from the standard inner product $\langle$,$\rangle on \mathbf{C}^{4}$, we can check that this complex almost contact metric structure $(u, v, U, V, G, H, J, g)$ is I-K normal. Thus we get a new example of a normal complex almost contact metric manifold. 


\section{A complex almost contact metric structure on $S^{4 m+3} \times S^{4 n+3}$}

We recall the 3-Sasakian structures on $M^{4 p+3}$.

DEFINITION 7.1. Let $M^{4 n+3}$ be a real (4n+3)-dimensional manifold. The 3-Sasakian structure on $M^{4 n+3}$ is a triple of Sasakian structures $\left\{\Phi_{i}, \xi_{i}, \eta_{i}\right\}_{i=1,2,3}$ on $M^{4 n+3}$ satisfying

$$
\begin{aligned}
& \Phi_{k}=\Phi_{i} \Phi_{j}-\eta_{j} \otimes \xi_{i}=-\Phi_{j} \Phi_{i}+\eta_{i} \otimes \xi_{j}, \\
& \eta_{i} \circ \Phi_{j}=\eta_{k}, \quad \eta_{i}\left(\xi_{j}\right)=\delta_{i j},
\end{aligned}
$$

where $\{i, j, k\}$ is one of the cyclic permutations of $\{1,2,3\}$. Define $M^{4 n+3}$ is called the 3Sasakian manifold if there exists a 3-Sasakian structure on it.

The typical example of 3-Sasakian manifold is $S^{4 n+3}$ obtained by taking as a hypersurface in the quaternion vector space $\mathbf{H}^{n+1}$. Each of three almost complex structures on $\mathbf{H}^{n+1}$ applied to the outer normal vector of the sphere gives a vector field $\xi_{i}, i=1,2,3$, on $S^{4 n+3}$. These three vector fields are orthogonal each other and give rise to the standard 3-Sasakian structure on $S^{4 n+3}$.

We show that 3-Sasakian structures on $S^{4 m+3}$ and $S^{4 n+3}$ induce a complex almost contact metric structure on $S^{4 m+3} \times S^{4 n+3}$. Let $\left\{\Phi_{i}^{m}, \xi_{i}^{m}, \eta_{i}^{m}\right\}_{i=1,2,3}$ and $\left\{\Phi_{i}^{n}, \xi_{i}^{n}, \eta_{i}^{n}\right\}_{i=1,2,3}$ be 3Sasakian structures on $S^{4 m+3}$ and $S^{4 n+3}$, respectively. We first define an almost complex structure on $S^{4 m+3} \times S^{4 n+3}$ by

$$
J_{m, n}(X, Y)=\left(\Phi_{1}^{m} X-\eta_{1}^{n}(Y) \xi_{1}^{m}, \Phi_{1}^{n} Y+\eta_{1}^{m}(X) \xi_{1}^{m}\right),
$$

where $(X, Y) \in T\left(S^{4 m+3} \times S^{4 n+3}\right)$. Since $J_{m, n}$ is integrable [16] (see also [6]), then $\left(S^{4 m+3} \times S^{4 n+3}, J_{m, n}\right)$ is a complex manifold. Moreover, it is also proved that the product space of two normal almost contact metric manifolds is a complex manifold with the above $J_{m, n}$. Next, we define a metric $g_{m, n}$ on $S^{4 m+3} \times S^{4 n+3}$ by

$$
\begin{aligned}
g_{m, n}\left((X, Y),\left(X^{\prime}, Y^{\prime}\right)\right)= & g_{m}\left(X, X^{\prime}\right)+\eta_{1}^{m}(X) \eta_{1}^{m}\left(X^{\prime}\right) \\
& +g_{n}\left(Y, Y^{\prime}\right)+\eta_{1}^{n}(Y) \eta_{1}^{n}\left(Y^{\prime}\right),
\end{aligned}
$$

where $g_{m}$ and $g_{n}$ are the associated metrics to 3-Sasakian structures on $S^{4 m+3}$ and $S^{4 n+3}$ respectively. It is easily checked that $g_{m, n}$ is a Hermitian metric with respect to $J_{m, n}$.

$X \in T M^{4 m+3}$ and $Y \in T M^{4 n+3}$ are decomposed to the subspace spanned by $\left\{\xi_{1}^{m}, \xi_{2}^{m}, \xi_{3}^{m}\right\},\left\{\xi_{1}^{n}, \xi_{2}^{n}, \xi_{3}^{n}\right\}$ and their orthogonal complements uniquely as follows.

$$
\left\{\begin{array}{l}
X=X_{0}+\eta_{1}^{m}(X) \xi_{1}^{m}+\eta_{2}^{m}(X) \xi_{2}^{m}+\eta_{3}^{m}(X) \xi_{3}^{m} \\
Y=Y_{0}+\eta_{1}^{n}(X) \xi_{1}^{n}+\eta_{2}^{n}(X) \xi_{2}^{n}+\eta_{3}^{n}(X) \xi_{3}^{n}
\end{array}\right.
$$


where $X_{0} \in \operatorname{Span}\left\{\xi_{1}^{m}, \xi_{2}^{m}, \xi_{3}^{m}\right\}^{\perp}, Y_{0} \in \operatorname{Span}\left\{\xi_{1}^{n}, \xi_{2}^{n}, \xi_{3}^{n}\right\}^{\perp}$. With this decomposition, we define the two type- $(1,1)$ tensors $G_{m, n}$ and $H_{m, n}$ by

$$
\begin{aligned}
& G_{m, n}(X, Y)=\left(\Phi_{2}^{m} X_{0}-\frac{\eta_{3}^{m}(X)-\eta_{3}^{n}(Y)}{2} \xi_{1}^{m}+\eta_{1}^{n}(Y) \xi_{2}^{m}+\eta_{1}^{m}(X) \xi_{3}^{m},\right. \\
& \left.\Phi_{2}^{n} Y_{0}-\frac{\eta_{2}^{m}(X)-\eta_{2}^{n}(Y)}{2} \xi_{1}^{n}-\eta_{1}^{m}(Y) \xi_{2}^{n}-\eta_{1}^{m}(X) \xi_{3}^{n}\right), \\
& \text { (19) } \quad H_{m, n}(X, Y)=J_{m, n} G_{m, n}(X, Y) \text {, }
\end{aligned}
$$

where $(X, Y) \in T\left(S^{4 m+3} \times S^{4 n+3}\right)$. We can check that $G$ and $H$ satisfy the condition to be a complex almost contact metric structure. Using the formula (18), we get

$$
\begin{aligned}
& G_{m, n}^{2}(X, Y) \\
= & \left(\left(\Phi_{2}^{m}\right)^{2} X_{0}-\eta_{1}^{m}(X) \xi_{1}^{m}+\frac{\eta_{2}^{m}(X)-\eta_{2}^{n}(Y)}{2} \xi_{2}^{m}-\frac{\eta_{3}^{m}(X)-\eta_{3}^{n}(Y)}{2} \xi_{3}^{m},\right. \\
& \left.\left(\Phi_{2}^{n}\right)^{2} Y_{0}-\eta_{1}^{n}(Y) \xi_{1}^{n}+\frac{\eta_{2}^{m}(X)-\eta_{2}^{n}(Y)}{2} \xi_{2}^{n}+\frac{\eta_{3}^{m}(X)-\eta_{3}^{n}(Y)}{2} \xi_{3}^{n}\right) \\
= & \left(-X_{0}-\eta_{1}^{m}(X) \xi_{1}^{m}-\eta_{2}^{m}(X) \xi_{2}^{m}-\eta_{3}^{m}(X) \xi_{3}^{m}\right. \\
& \left.-Y_{0}-\eta_{1}^{n}(Y) \xi_{1}^{n}-\eta_{2}^{n}(Y) \xi_{2}^{n}-\eta_{3}^{n}(Y) \xi_{3}^{n}\right) \\
& +\frac{\eta_{2}^{m}(X)+\eta_{2}^{n}(Y)}{2}\left(\xi_{2}^{m}, \xi_{2}^{n}\right)+\frac{\eta_{3}^{m}(X)+\eta_{3}^{n}(Y)}{2}\left(\xi_{3}^{m}, \xi_{3}^{n}\right),
\end{aligned}
$$

where $(X, Y) \in T\left(S^{4 m+3} \times S^{4 n+3}\right)$. Here we define 1-forms $u_{m, n}, v_{m, n}$ and dual orthonormal vector fields $U_{m, n}, V_{m, n}$ which satisfy Definition 2.2. by

$$
\left\{\begin{array}{l}
u_{m, n}=\frac{1}{\sqrt{2}}\left(\eta_{3}^{m}+\eta_{3}^{n}\right), \quad v_{m, n}=\frac{1}{\sqrt{2}}\left(\eta_{2}^{m}+\eta_{2}^{n}\right), \\
U_{m, n}=\frac{1}{\sqrt{2}}\left(\xi_{3}^{m}, \xi_{3}^{n}\right), \quad V_{m, n}=\frac{1}{\sqrt{2}}\left(\xi_{2}^{m}, \xi_{2}^{n}\right) .
\end{array}\right.
$$

With these elements, we get

$$
G_{m, n}^{2}(X, Y)=-(X, Y)+u_{m, n}(X, Y) \otimes U+v_{m, n}(X, Y) \otimes V .
$$

Moreover, by (17), (18) and (20), we have

$$
\begin{gathered}
J_{m, n} G_{m, n}(X, Y)=-G_{m, n} J_{m, n}(X, Y) \\
=\left(\Phi_{3}^{m} X_{0}+\frac{\eta_{2}^{m}(X)-\eta_{2}^{n}(Y)}{2} \xi_{1}^{m}-\eta_{1}^{m}(X) \xi_{2}^{m}+\eta_{1}^{n}(Y) \xi_{3}^{m},\right. \\
\left.\Phi_{3}^{n} Y_{0}-\frac{\eta_{3}^{m}(X)-\eta_{3}^{n}(Y)}{2} \xi_{1}^{n}+\eta_{1}^{m}(X) \xi_{2}^{n}-\eta_{1}^{n}(Y) \xi_{3}^{n}\right) . \\
g_{m, n}\left(G_{m, n}(X, Y),\left(X^{\prime}, Y^{\prime}\right)\right)=-g_{m, n}\left((X, Y), G_{m, n}\left(X^{\prime}, Y^{\prime}\right)\right)
\end{gathered}
$$




$$
\begin{aligned}
= & g_{m}\left(\Phi_{2}^{m} X_{0}, X_{0}^{\prime}\right)+g_{n}\left(\Phi_{2}^{n} Y_{0}, Y_{0}^{\prime}\right)+\eta_{1}^{m}(X)\left(\eta_{3}^{m}\left(X^{\prime}\right)-\eta_{3}^{n}\left(Y^{\prime}\right)\right) \\
& -\eta_{1}^{m}\left(X^{\prime}\right)\left(\eta_{3}^{m}\left(X^{\prime}\right)-\eta_{3}^{n}\left(Y^{\prime}\right)\right)+\eta_{1}^{n}(Y)\left(\eta_{2}^{m}\left(X^{\prime}\right)-\eta_{2}^{n}\left(Y^{\prime}\right)\right) \\
& -\eta_{1}^{n}\left(Y^{\prime}\right)\left(\eta_{2}^{m}(X)-\eta_{3}^{n}(Y)\right) .
\end{aligned}
$$

Note that the second Betti number of compact Kähler manifolds is not zero, and Künneth formula shows [9]

$$
H^{2}\left(S^{4 m+3} \times S^{4 n+3}\right) \cong \bigoplus_{i+j=2} H^{i}\left(S^{4 m+3}\right) \otimes H^{j}\left(S^{4 n+3}\right) \cong\{0\} .
$$

Since $S^{4 m+3} \times S^{4 n+3}$ admits no Kähler structure, this structure is not I-K normal.

THEOREM 7.2. The complex almost contact metric structure on $S^{4 m+3} \times S^{4 n+3}$ $\left(G_{m, n}, H_{m, n}, J_{m, n}, u_{m, n}, v_{m, n}, U_{m, n}, V_{m, n}, g_{m, n}\right)$ given by (15), (16), (18), (19) and (21) is not I-K normal.

\section{References}

[1] C. Baikoussis, D. Blair and F. Gouli-Andreou, Holomorphic Legendre curves in the complex Heisenberg group, Bull. Inst Math. Acad. Sinica 26 (1998), 179-194.

[2] D. BLAIR, Contact Manifolds in Riemannian Geometry, Lecture Notes in Math. 509, Springer, Berlin, 1976.

[3] D. BLAIR, Riemannain Geometry of Contact and Symplectic Manifolds second edition, Progress in Mathematics 203, Birkhäuser, 2010.

[4] W. M. Воотнв , Homogeneous complex contact manifolds, Proc. Sympos. Pure Math III, Amer. Math. Soc., Providence, 1961, 144-154.

[ 5 ] W. M. Bоотнвy, A note on homogeneous complex contact manifolds, Proc. Amer. Math. Soc. 10 (1962), 276-280.

[6] E. CALABI and B. EcKmann, A class of compact complex manifolds which are not algebraic, Ann. of Math. $\mathbf{5 8}(1953)$, 494-500.

[ 7 ] M. DJoriC and M. OKumura, CR Submanifolds of Complex Projective Space, Developments in Mathematics 19, Springer, Berlin, 2009.

[ 8 ] B. Foreman, Variational problems on complex contact manifolds with applications to twistor space theory, Thesis, Michigan State University, 1996.

[9] P. GRIFFITHS and J. HARRIS, Principles of Algebraic Geometry, Wiley Classics Library, John Wiley \& Sons Inc., New York 1994, Reprint of the 1978 original.

[10] S. IshiHARA and M. Konishi, Real contact 3-structure and complex contact structure, Southeast Asian Bull. Math. 3 (1979), 151-161.

[11] S. Ishihara and M. Konishi, Complex almost contact manifolds, Kōdai Math. J. 3 (1980), 385-396.

[12] S. Ishihara and M. Konishi, Complex almost contact structures in a complex contact manifold, Kōdai Math. J. 5 (1982), 30-37.

[13] S. Kobayashi, Remarks on complex contact manifolds, Proc. Amer. Math. Soc. 10 (1959), 164-167.

[14] S. KobAYASHI, Topology of positively pinched Kaehler manifolds, Tôhoku Math. J. 15 (1963), 121-139.

[15] B. KorkmaZ, Normality of complex contact manifolds, Rocky Mountain J. of Math. 30 (2000), 1343-1380.

[16] A. Morimoto, On normal almost contact structures, J. Math. Soc. Japan 15 (1963), 420-436. 
Present Address:

DePartment of Mathematics,

KeIO University,

HiYOSHI, KOHOKU-KU, YOKOHAMA 223-8532, JAPAN.

e-mail: imadddjp@gmail.com 